Case Report

Oopen Access

A) CrossMark

\title{
Severe acute myocarditis due to scorpion envenoming: unusual presentation in adults
}

\begin{abstract}
Scorpion envenomation (SE) represents a major public health issue, since more than one million cases occur every year, especially in tropical, subtropical and sub-Saharan areas. Clinical presentation varies from pain at site of sting to severe generalized intoxication. Cardiac involvement, usually reversible within the first week post-sting, has been reported with different degrees of acute left ventricle (LV) dysfunction. Despite cardiac magnetic resonance (CMR) imaging is recommended, when available, transthoracic echocardiogram (TTE) still remains an excellent tool for diagnostic and follow-up assessment. We report herein an interesting and unusual case of prompt recovery of severe acute myocarditis secondary to SE in a young woman.
\end{abstract}

Keywords: acute myocarditis, scorpion envenoming
Volume 13 Issue 3 - 2020

Glenda Alves de Sá,' Cássia Souza FVale,' Marcos Danillo P Oliveira ${ }^{2}$

'Department of Cardiology, Hospital Regional de São José dos Campos, Brazil

${ }^{2}$ Department of Interventional Cardiology, Hospital São Paulo, Brazil

Correspondence: Marcos Danillo P Oliveira, Department of Interventional Cardiology, Hospital São Paulo, Escola Paulista de Medicina, Universidade Federal de São Paulo, São Paulo, SP, Brazil,Email mdmarcosdanillo@gmail.com

Received: April 22, 2020 | Published: June 22, 2020
Abbreviations: SE, scorpion envenomation; LV, left ventricle; CMR, cardiac magnetic resonance; TTE, transthoracic echocardiogram; ARF, acute respiratory failure; LVEF, LV ejection fraction

\section{Case report}

A 21-year-old healthy woman presented to emergency department complaining of severe gluteus pain and progressive dyspnea about one hour after domestic accidental "black scorpion" sting. Despite early scorpion antivenom administration, advanced airway and intensive critical care were promptly required, due to refractory acute respiratory failure (ARF), endorsed by a critical $\mathrm{PaO} 2 /$ $\mathrm{FiO} 2$ of 48 . Chest computed tomography revealed diffuse bilateral infiltrates and air bronchograms compatible with acute respiratory distress syndrome. Cardiac troponin levels continuously rose. Initial transthoracic echocardiogram (TTE) revealed diffuse hypokinesia causing moderate left ventricle (LV) systolic dysfunction - rest LV ejection fraction (LVEF) of 0.39 (Simpson's method) (Figure 1). Inotrope at low doses (dobutamine $5 \mathrm{mcg} / \mathrm{kg} / \mathrm{min}$ ) was then initiated. Follow-up (ninth day) TTE (Figure 2) and cardiac magnetic resonance (CMR) imaging endorsed reversal of initial contractile dysfunction. At eleventh day patient was uneventfully discharged home.

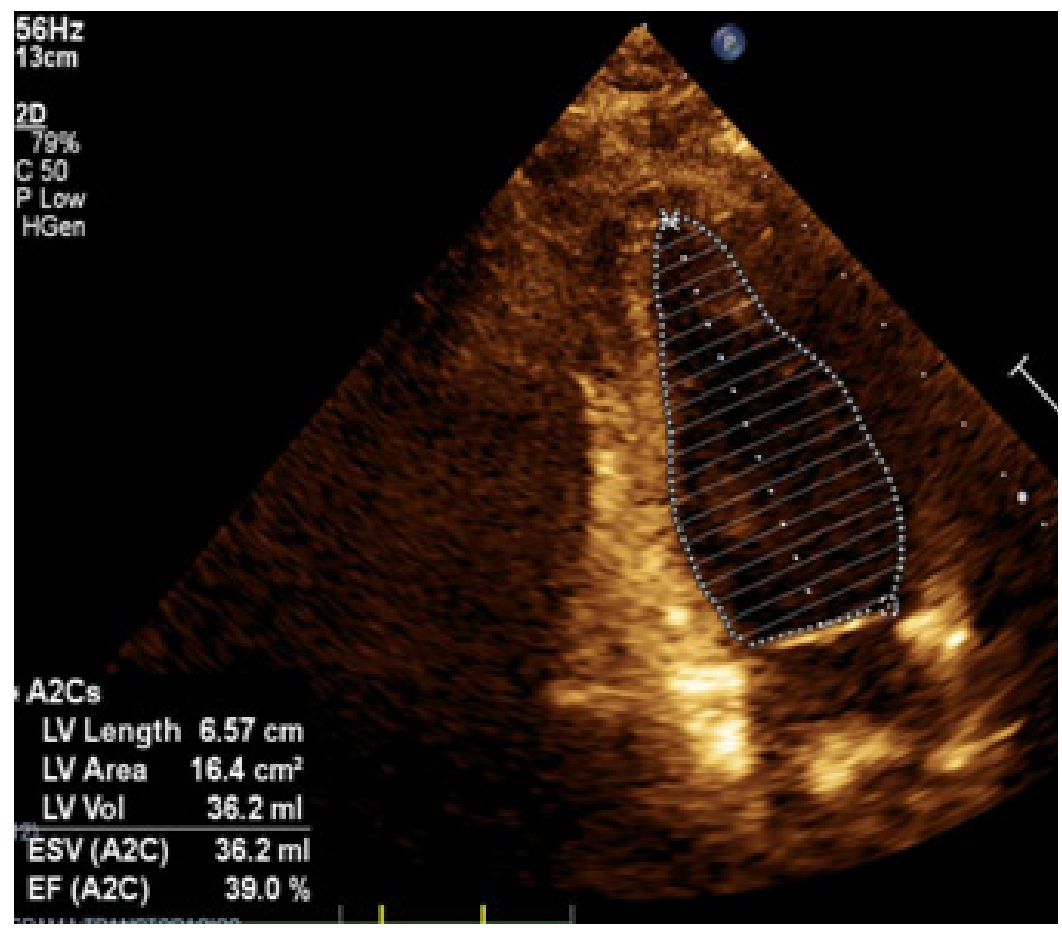

Figure I Baseline transthoracic echocardiogram showing left ventricular ejection fraction of 0.39 (Simpson's method) due to diffuse hypokinesia. 


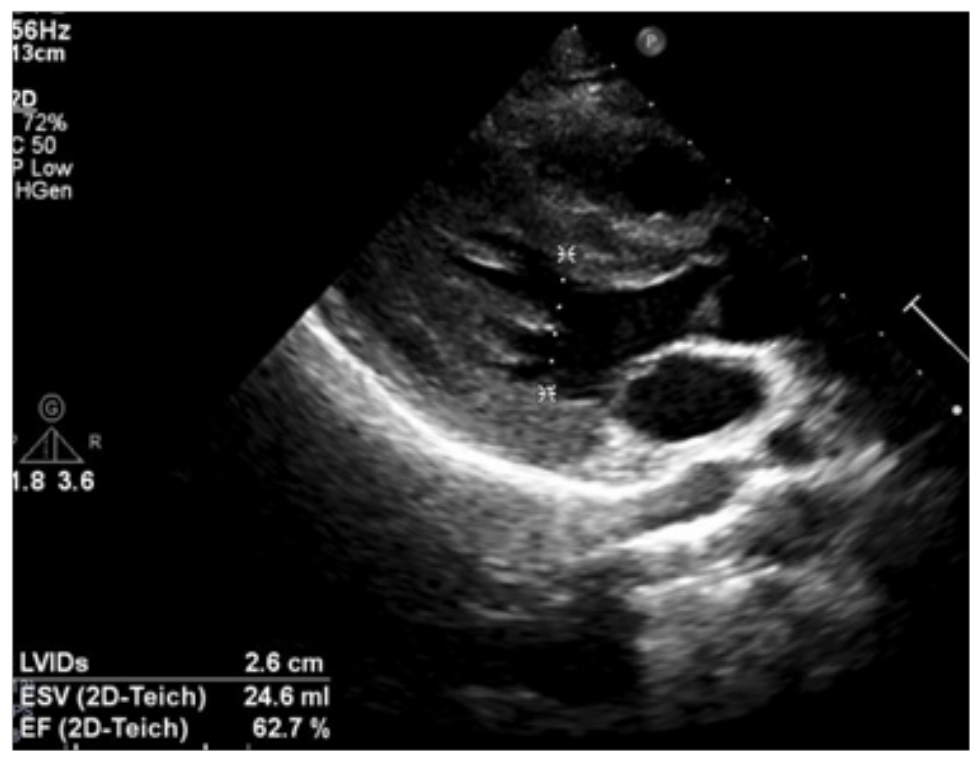

Figure 2 Control transthoracic echocardiogram showing left ventricular ejection fraction of 0.63 (Teichholz's method) due to recovered myocardial contractility.

\section{Discussion}

Scorpion envenomation (SE) represents a major public health issue, since each year more than one million cases occur, especially in tropical, subtropical and sub-Saharan areas. ${ }^{1}$ Main toxic effects of scorpion venom are due to its $\alpha$-toxins inhibition of voltagegated sodium channel inactivation, resulting in prolonged neuronal depolarization. ${ }^{2,3}$

Clinical presentation varies from local pain at site of sting to severe generalized intoxication, mainly due to venom induced cholinergic and adrenergic effects., ${ }^{4,5}$ Acetylcholine release induces: myosis, bradyarrhythmias, arterial hypotension, increased lachrymal, nasal, salivary, pancreatic, gastric and bronchial secretions, diaphoresis, tremors, piloerection and muscle spasms. In turn, catecholamine releasing induces mydriasis, tachyarrhythmias, arterial hypertension, acute pulmonary edema, cardiac failure and shock. ${ }^{5}$

Cardiac involvement, usually reversible within the first week post-sting, has been mainly attributed to adrenergic firing induced by scorpion toxins. Catecholamine-mediated cardiac damage seems to be multifactorial: relative hypoxia caused by increased heart rate; coronary spasm and microcirculation vasoconstriction; and direct toxic effect on myocardial cells through increase in intracellular calcium..$^{4-6}$

Different degrees of acute LV systolic dysfunction with abnormal global or regional mobility occurring soon after the sting can be observed. This may be accompanied by increased levels of cardiac biomarkers and electrocardiographics or TTE changes, without following anatomic distribution of coronary arteries. ${ }^{7-9} \mathrm{LV}$ systolic dysfunction may occurs with cardiac chambers dilatation and mitral valve regurgitation. Involvement of the right ventricle has been reported in up to $50 \%$ of patients with serious envenoming..$^{10} \mathrm{TTE}$ changes may be present as early as the first few hours after the sting, thus being an important tool for diagnosis and prognosis. CMR imaging is also useful for confirming recovery, as in the present case, and especially for detection of irreversible myocardial damage and dilated cardiomyopathy delayed development.,11

\section{Conclusion}

Despite more common in childhood, severe forms of SE can be life-threatening also in adults and should therefore be promptly recognized and treated, in order to avoid unfavorable outcomes.

\section{Acknowledgments}

None.

\section{Conflicts of interest}

Author declares that there are no conflicts of interest.

\section{Funding}

None.

\section{References}

1. Isbister GK, Bawaskar HS. Scorpion envenomation. $N$ Engl J Med. 2014;371:457-463.

2. Abroug F, Souheil E, Ouanes I, et al. Scorpionrelatedcardiomyopathy:Clinical characteristics, pathophysiology, and treatment. Clin Toxicol. 2015;53(6):511-518.

3. Ismail $\mathrm{M}$, Asaad N, Al Suwaidi $\mathrm{J}$, et al. Acute myocarditis and pulmonary edema due to scorpion sting. Glob Cardiol Sci Pract. 2016;2016(1):e201610.

4. Bucaretchi F, Fernandes LCR, Fernandes CB, et al. Clinical consequences of Tityus bahiensis and Tityus serrulatus scorpion stings in the region of Campinas, southeastern Brazil. Toxicon. 2014;89:17-25.

5. Cupo P, Clinical update on scorpion envenoming. Rev Soc Bras de Medicina Tropical. 201548(6):642-649.

6. Gueron M, Stern J, Cohen W. Severe myocardial damage and heart failure in scorpion sting. Report of five cases. Amer J Cardiol. 1967;19(5):719726.

7. Gueron M, Margulis G, Sofer S. Echocardiographic and radionuclide angiographic observations following scorpion envenomation by Leiurus quinquestriatus. Toxicon. 1990;28(90:1005-1009. 
8. Amaral CF, Lopes JA, Magalhães RA, et al. Electrocardiographic, enzymatic and echocardiographic evidence of myocardial damage after Tityus serrulatus scorpion poisoning. Am J Cardiol. 1991;67:655-657.

9. Hering SE, Jurca M, Vichi FL, Azevedo-Marques MM, Cupo P. "Reversible cardiomyopathy" in patients with severe scorpion envenoming by Tityus serulatus:evolution of enzymatic, electrocardiographic and echocardiographic alterations. Ann Trop Paediatr. 1993;13(2):173-182.
10. Nouira S, Abroug F, Haguiga $\mathrm{H}$, et al. Right ventricular dysfunction following severe scorpion envenomation. Chest. 1995;108:682-687.

11. Lonati D, Locatelli CA, Sabri S, et al. Cardiac magnetic resonance study of scorpion toxic myocarditis. QJM. 2017;110(2):113-114. 